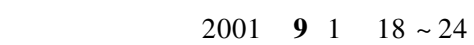

Biodiversity Science

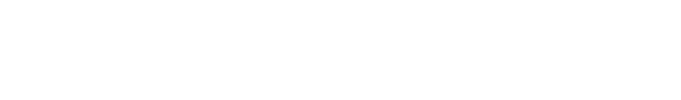

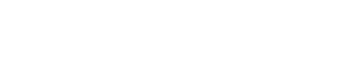

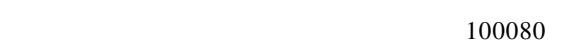

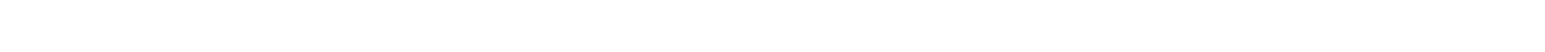

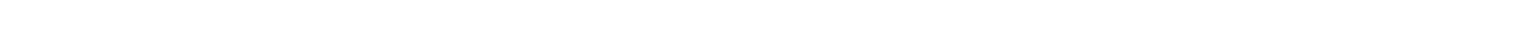

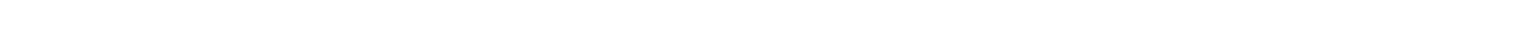

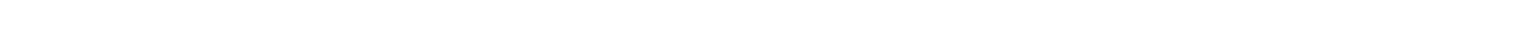

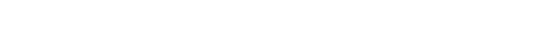

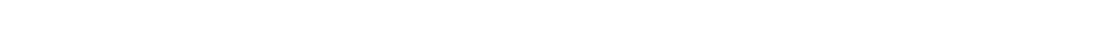

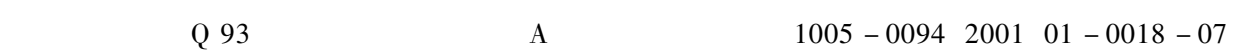

\section{Diversity of prokaryotic microorganisms}

DONG Xiu-ZhuftHONG Jun-Hua

Institute of Microbiology $\mathbf{f}$-Chinese Academy of Sciences $\mathbf{E - B e i j i n g ~} 100080$

\begin{abstract}
M i c$ roorganisms are an important group of organism mainly performing catabolic metabolism $€ \neg$ which display extreme abundance in biodiversity. Howeverf-due to their microscopic sizesf-especially prokaryotes with simple unicellular structuref-their asexual propagation $f$ their rapid replication and the lack of base line inventory of their groups and quantity $f$ the biodiversity of microorganisms has so far not been studied thoroughly and has been paid little attention compared with macro-organisms. In the present paperfthe biodiversity of microorganisms is reviewed briefly $f_{-w i t h}$ consideration of the following aspects $\mathfrak{}^{\circ}$ species diversity $\mathbf{f}$-liversity of phylogenetic lines $f_{p}$ hysiological diversity and genetic diversity. The aim is to improve awareness of investigation of microorganism resourcesf also in society as a whole.
\end{abstract}

Key wordsf diversity $\mathbf{f}$ genetic diversity.

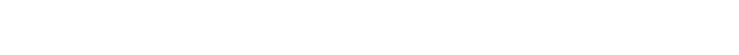

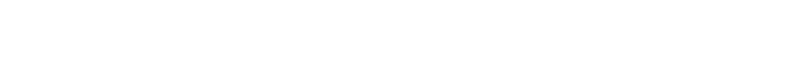

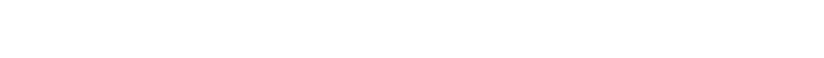
AE生âó

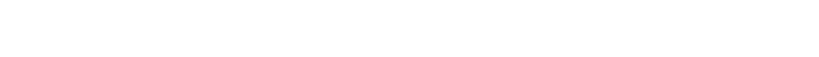

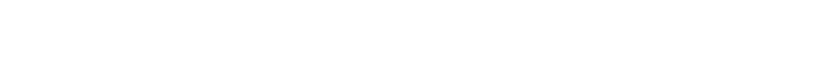

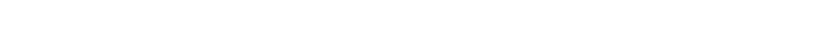

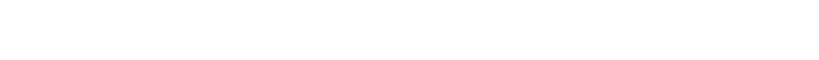

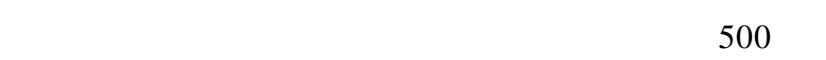

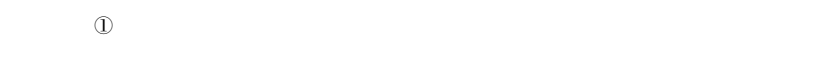

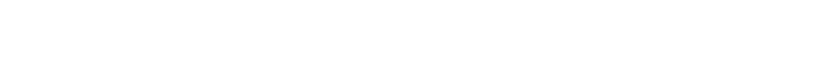

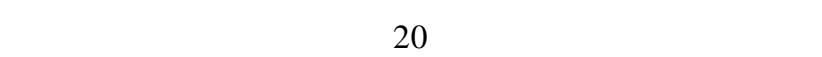

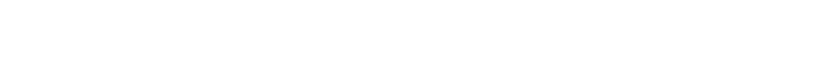

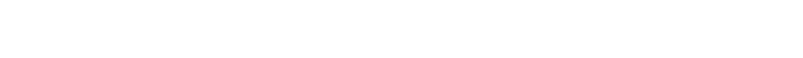

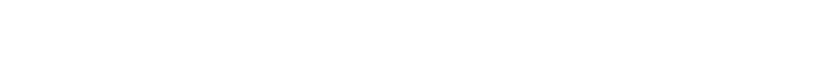

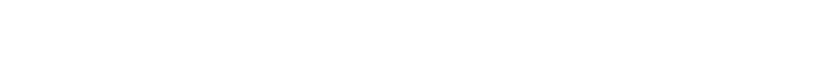

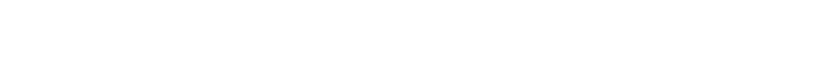

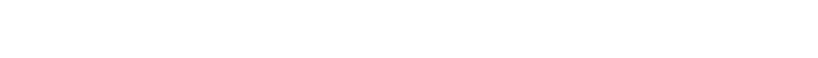

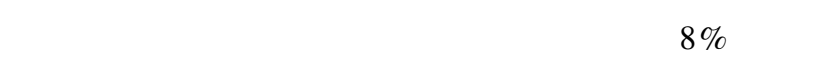

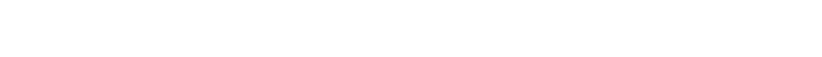

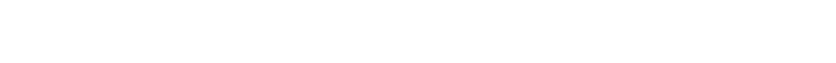

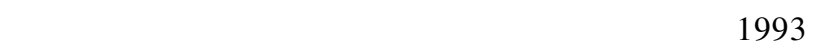




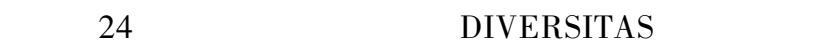

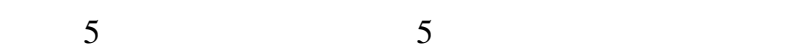

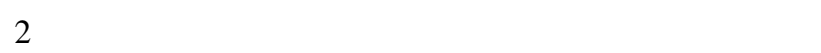

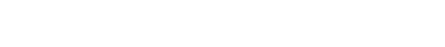

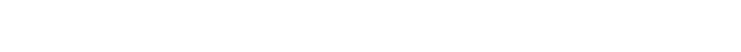

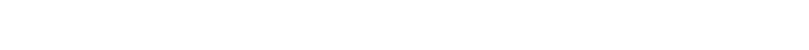

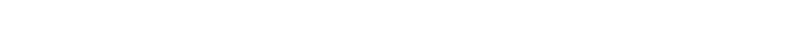

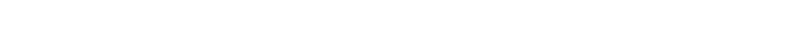

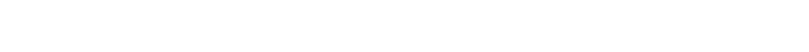
Ố $\mu \ddot{A}^{1} \varnothing \times \phi_{i} \mp$

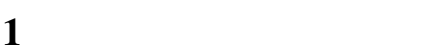

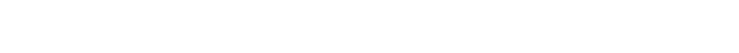

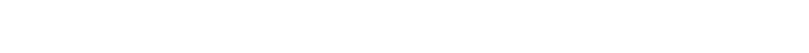

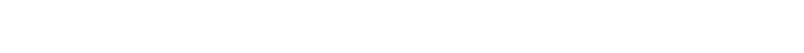

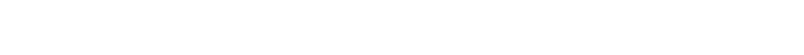

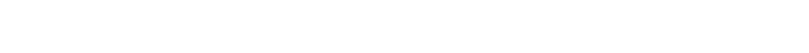

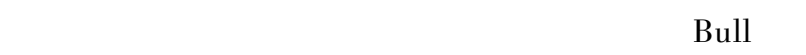

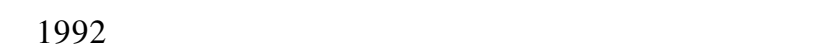

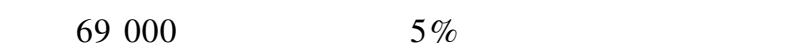

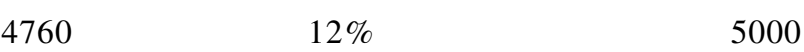

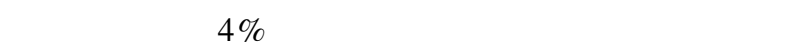

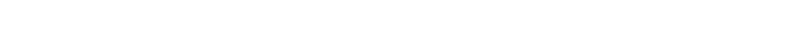

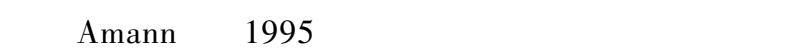

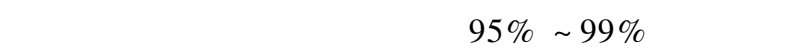

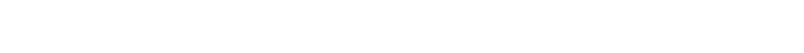

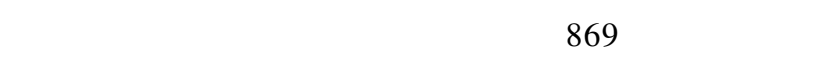

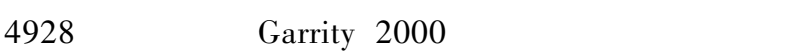

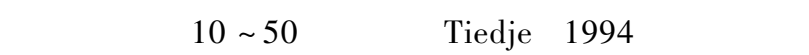
Éýxö ài çoî i $\mathrm{f}$

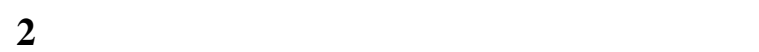

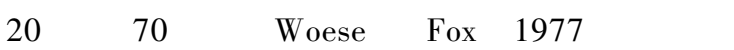

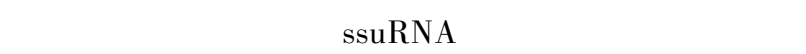

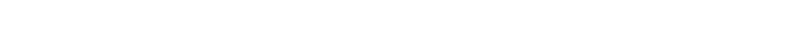

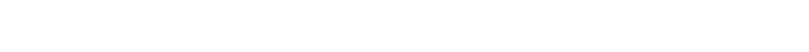

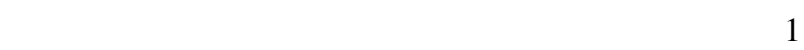

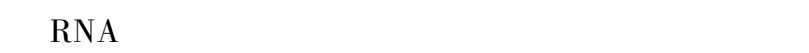

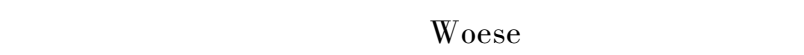

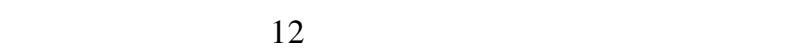

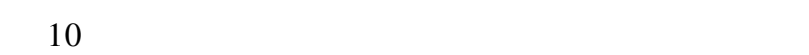

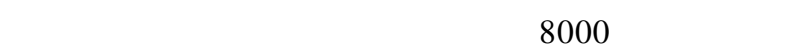

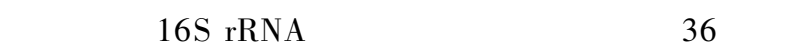

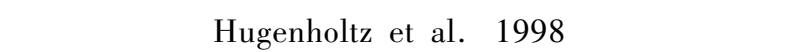

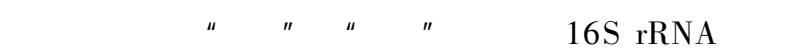

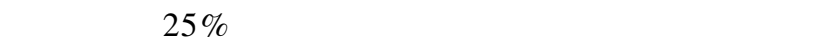

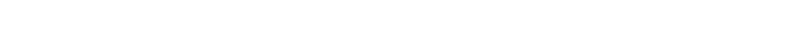

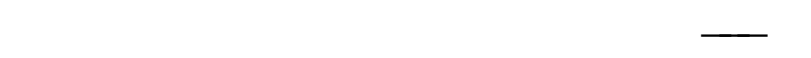

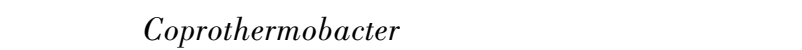

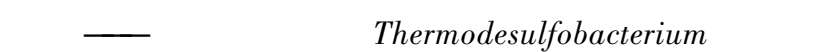

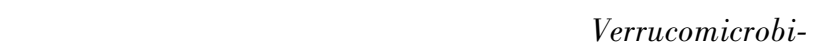

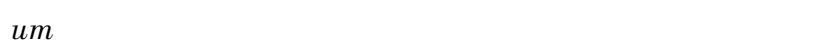
È-

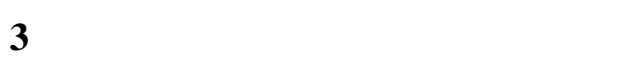

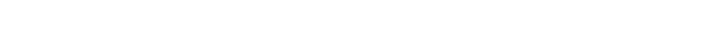

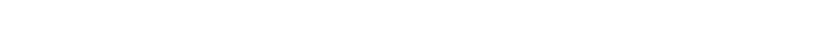

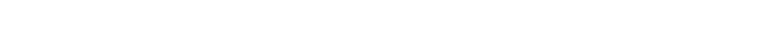

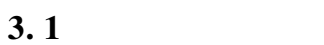

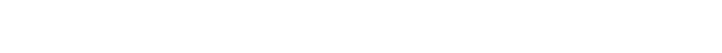

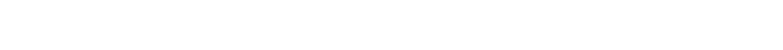

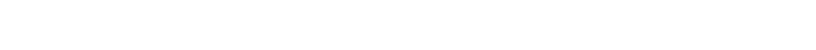

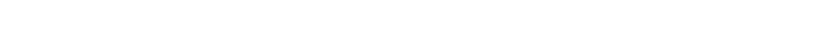

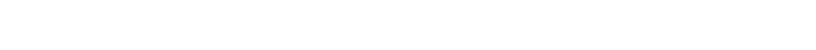

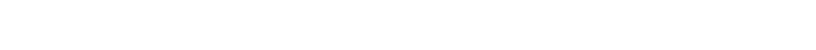

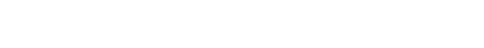

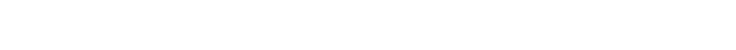

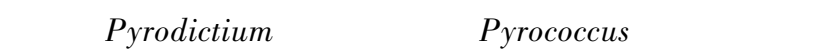

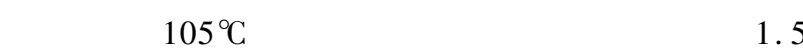

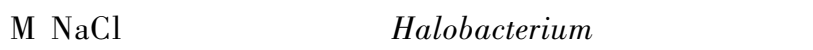

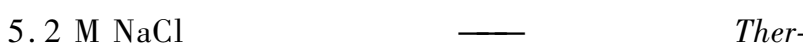

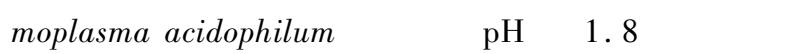

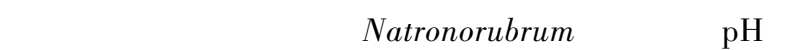
11 if

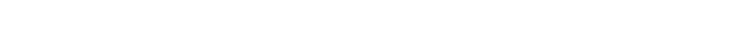

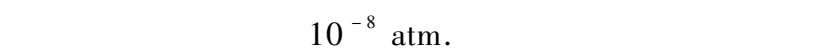

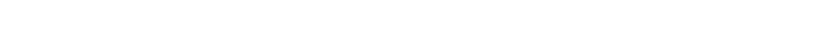

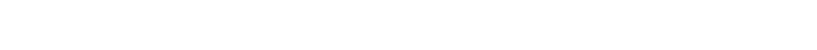

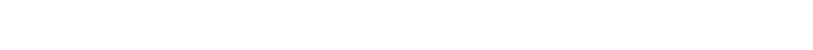

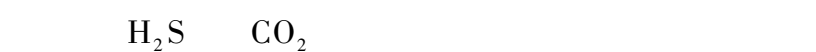

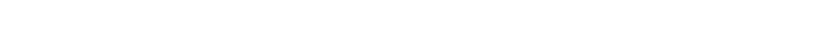
Îï Î p. "ì ǽúuÄi $f$

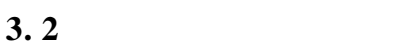

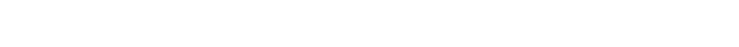

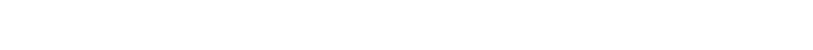

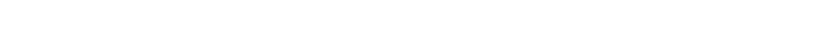

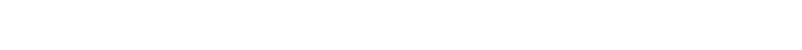
Ờ ¿Éi a Î

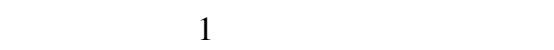




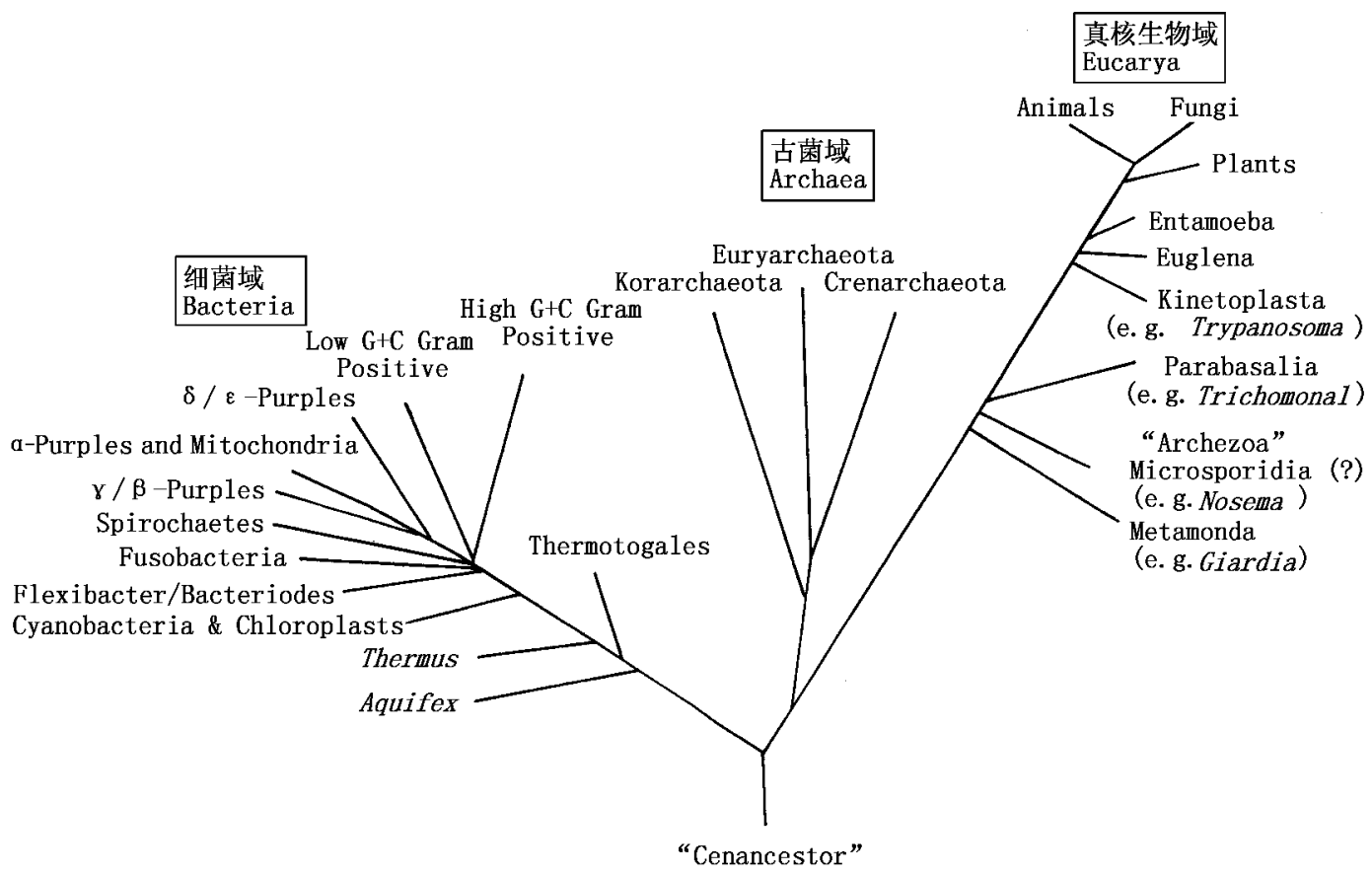

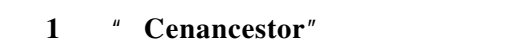

Fig. 1 Schematic drawing of a life tree rooting in ${ }^{\circ}$ Cenancestor \pm

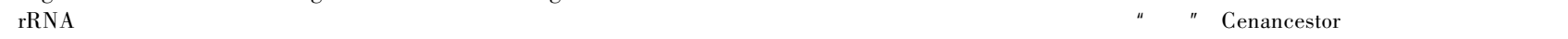

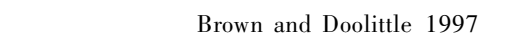

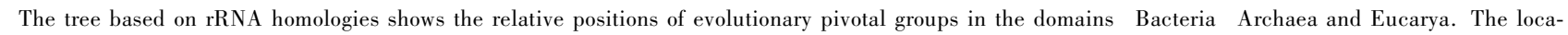

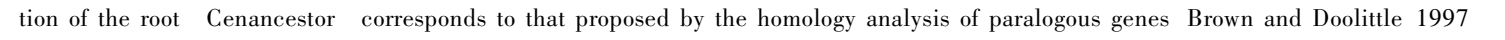

\section{培养的 \\ 未培养的 \\ cultivated uncultivated}

\section{变型菌纲}

Proteobacteria

放线杆菌

Actinobacteria

低GC革兰氏阳性菌

Low $\mathrm{G}+\mathrm{C}$ gram positive

噬纤维菌

Cytophagales

\section{酸杆菌}

Acidobacterium

状微菌

Verrucomicrobia

绿色非硫细菌

Green non-sulfur bacteria

0P11

$\begin{array}{lllll}100 & 50 & 0 & 50 & 100\end{array}$

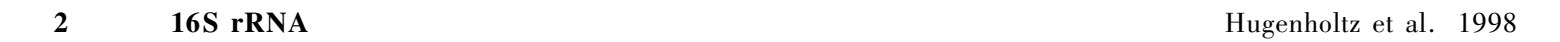

Fig. 2 Relative representation in different bacterial divisions of 16S rRNA sequences from cultivated and uncultivated organismsf" Hugenholtz et al. $£+998 £ @$ 


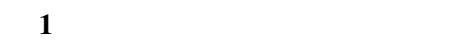

Table 1 Metabolic types and the representatives of bacteria

\begin{tabular}{|c|c|c|c|c|}
\hline & \multicolumn{2}{|c|}{$\begin{array}{c}\text { o } \tilde{A} \tilde{N} \sigma \\
\text { Aerobic }\end{array}$} & \multicolumn{2}{|c|}{ 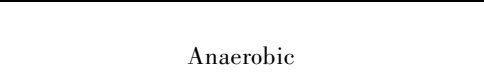 } \\
\hline & 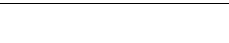 & ĆĐ\úó Ñø & Î p»úó Ñø & ĆĐ»úণ́ Ñø \\
\hline${ }^{1}$ âÄƯ̛̈́́ Ñø & ÀnẦ İ , 3র́ & ÎP & Ầ Á̀̀, Ë3晌 & ㅇ̀ İ , 3/র́ \\
\hline Phototrophic & Blue-green bacteria & None & Chlorobium & Rhodobacter \\
\hline » ÄÜỨ Ñø & 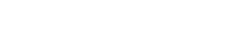 & 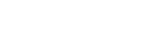 & 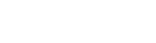 & 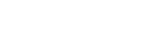 \\
\hline
\end{tabular}

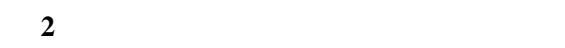

Table 2 Relationships between microorganisms and other lives

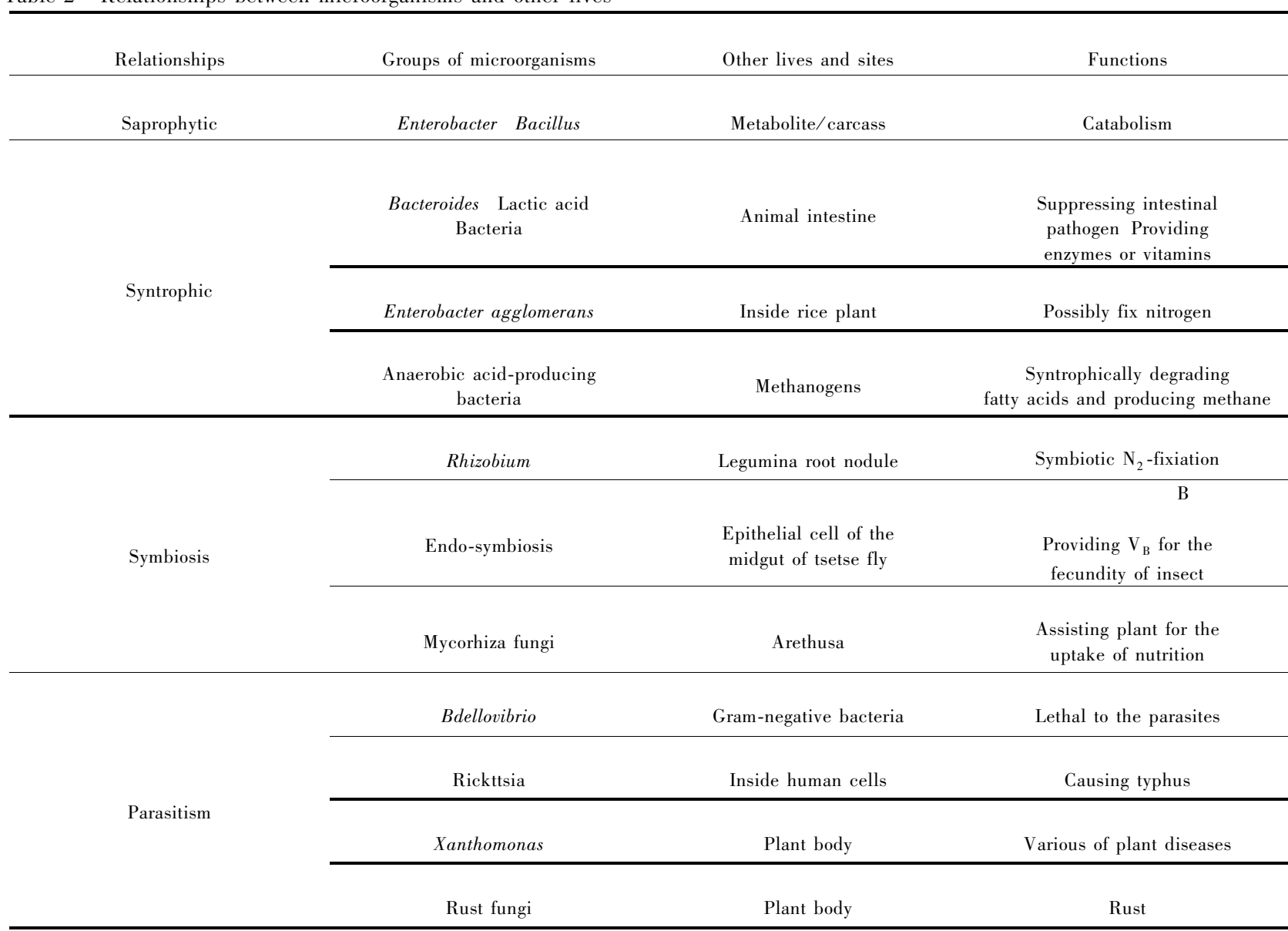

İ , 3/án

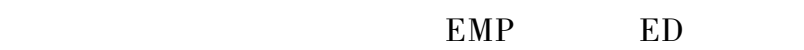

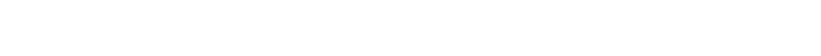

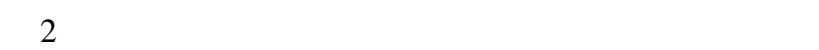

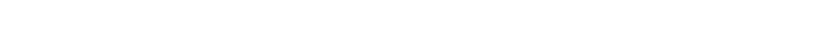

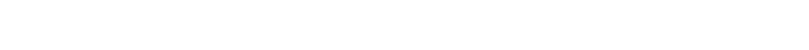

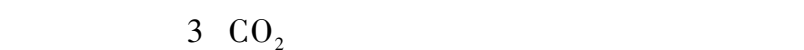

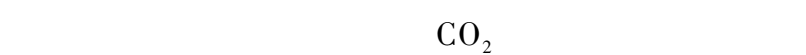

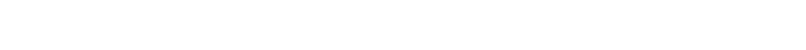

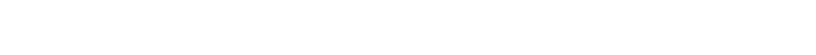
ËáÑ » ¡ $€$

ËùGXXÔÈ»

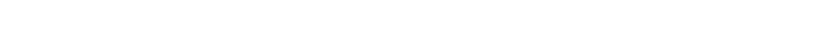

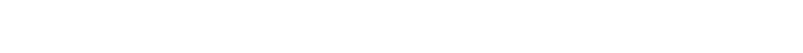
oï îï $\mu \dot{E}_{i} £$

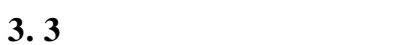

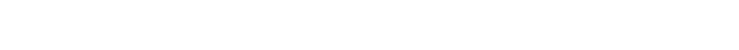

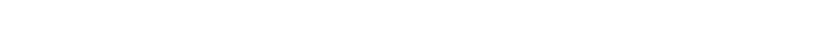




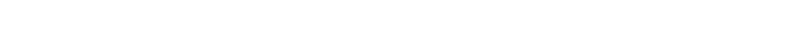

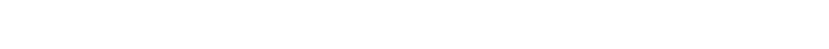

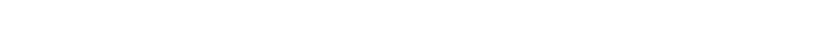

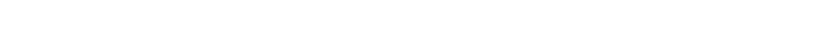

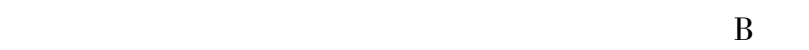

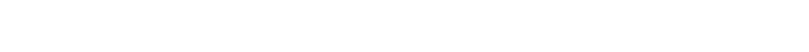

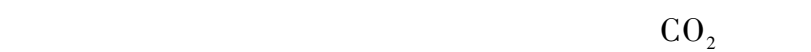

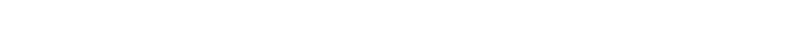

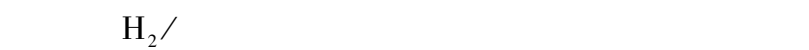

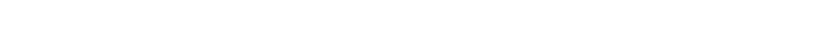

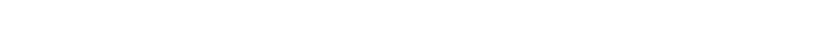

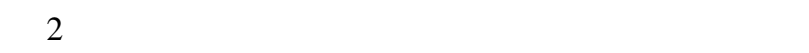

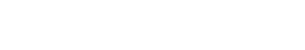

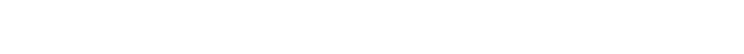

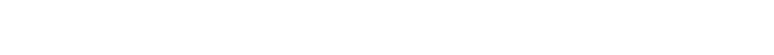

\section{4 Î đÉúîi}

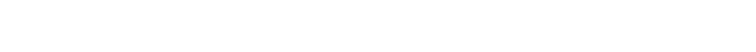

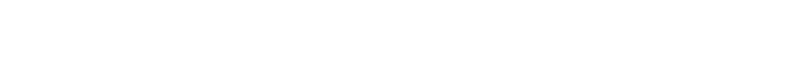
oÜ’ó

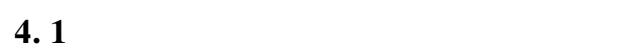

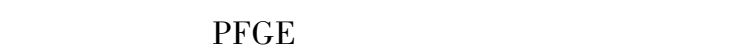

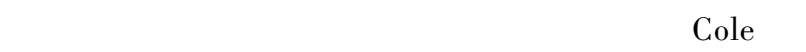

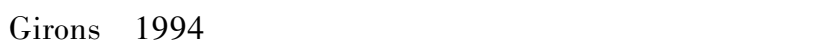

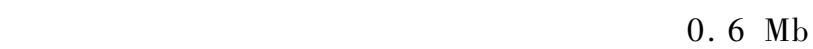

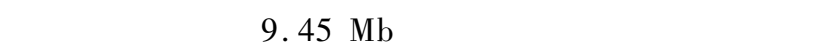

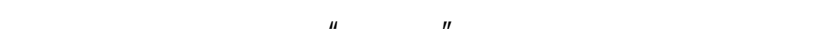

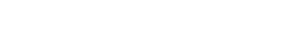

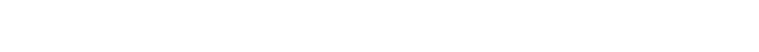

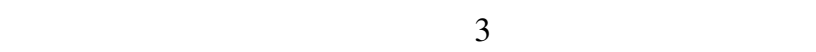

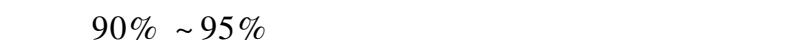

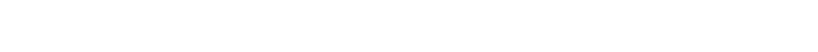

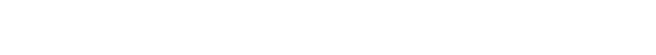

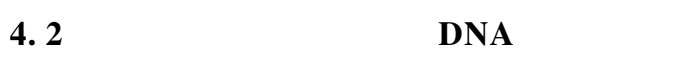

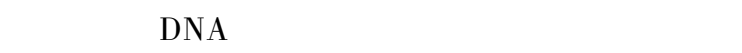

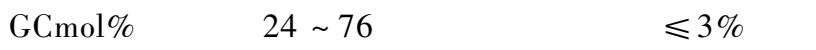
ÄÚ $\mu \ddot{A}^{2} i ̂$ o-Áti i fÁ ĺ

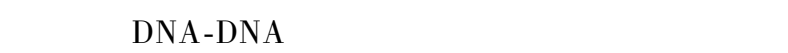

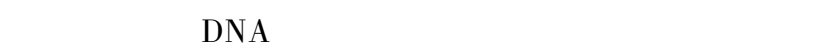

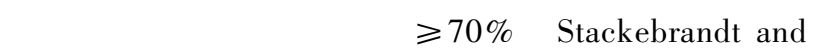

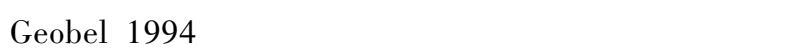

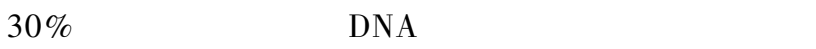

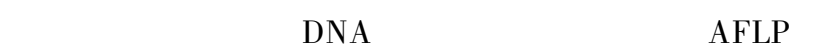

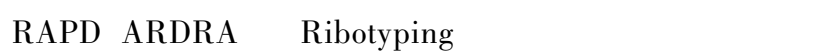

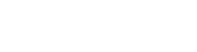

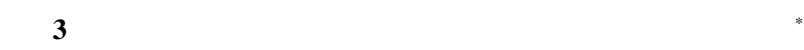

Table 3 Current predictions of approximate gene number and genome size in organisms in different evolutionary lineages

\begin{tabular}{|c|c|c|c|}
\hline & 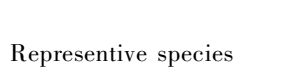 & $\begin{array}{l}\text { »ùÒ̀̇̂́ÿëé } \\
\text { gene number }\end{array}$ & $\begin{array}{l}\text { »uัOò̀xé'óĐfE" Mbf@ } \\
\text { genome size }\end{array}$ \\
\hline Ô oËÉúîï & Mycoplasma genitalium & 473 & 0.58 \\
\hline Prokaryota & Haemophilus influenzae & 1760 & 1.83 \\
\hline & Escherichia coli & 4100 & 4.7 \\
\hline & Myxococcus xanthus & 8000 & 9.45 \\
\hline Õß伯 & Saccharomyces cerevisiae & 5800 & 13.5 \\
\hline Protozoa & Oxytricha similis & 12000 & 600 \\
\hline 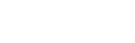 & Drosophila melanogaster & 12000 & 650 \\
\hline $\begin{array}{l}\text { Arthropoda } \\
\text { ï } \beta^{3} \not \mathbb{A}^{-} \mid i ̂ i\end{array}$ & Caenorhabditis elegans & 14000 & 100 \\
\hline $\begin{array}{l}\text { Nematoda } \\
\text { É İ İ åq-îï } \\
\text { Mollusca }\end{array}$ & Loligo pealii & $>35000$ & 2700 \\
\hline & Mus musculus & 70000 & 3300 \\
\hline & Homo sapiens & 70000 & 3300 \\
\hline Ölî & Nicotiana tabacum & 43000 & 4500 \\
\hline Plantae & Arabidopsis thaliana & $16000 \sim 33000$ & $70 \sim 145$ \\
\hline
\end{tabular}




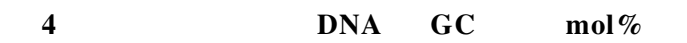

Table 4 DNA GC contentsf" mol\% $£ @$ n representative bacteria groups

\begin{tabular}{|c|c|c|c|}
\hline 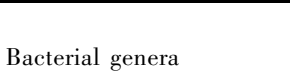 & $\begin{array}{c}\text { GC o-Áf́" mol\% f® } \\
\text { GC content }\end{array}$ & 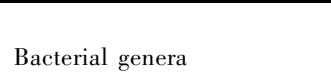 & 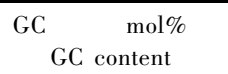 \\
\hline Ëó3㖧̂̂ Clostridium & $24 \sim 43$ & 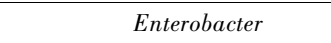 & $52 \sim 59$ \\
\hline 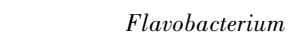 & $30 \sim 42$ & 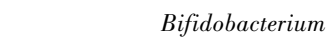 & $57 \sim 65$ \\
\hline 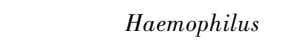 & $38 \sim 42$ & 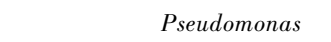 & $58 \sim 70$ \\
\hline 土äも行㖧ô Proteus & $38 \sim 42$ & 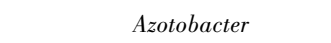 & $63 \sim 66$ \\
\hline 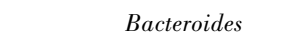 & $40 \sim 45$ & Á $^{\prime} \tilde{A}^{1}$ 3/র̂Ê̂ Streptomyces & $69 \sim 73$ \\
\hline
\end{tabular}

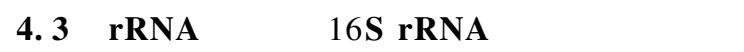

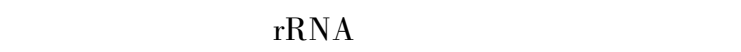

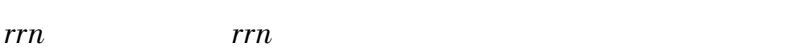
Ö̃̃ Ö̋̈oำ

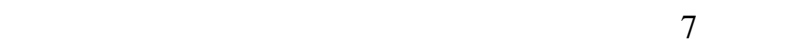

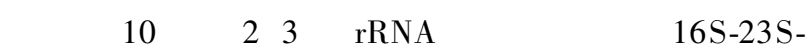

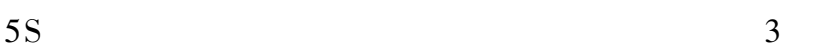

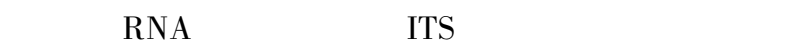

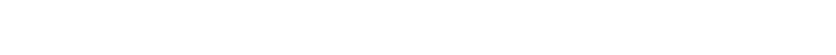

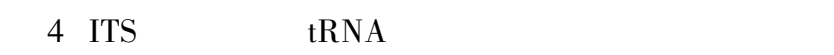

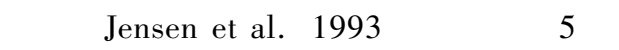

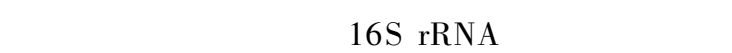

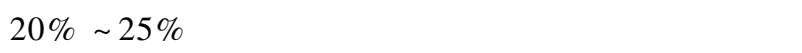

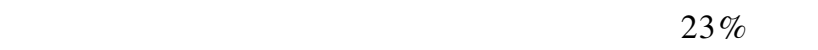

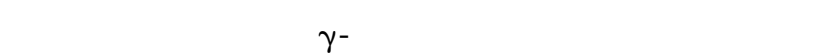

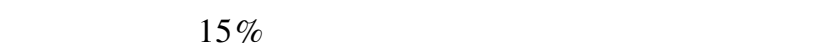
'ï $3 \%$ if

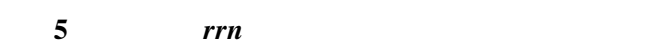

Table 5 Copy number and linkage of rrn locus of bacteria

\begin{tabular}{|c|c|c|}
\hline $\begin{array}{l}\text { İ , 3/6́ } \\
\text { Bacteria }\end{array}$ & 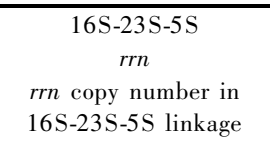 & 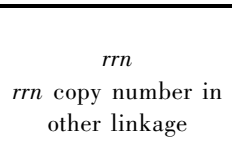 \\
\hline Acholeplasma laidlawii & & 2 \\
\hline Anabaena sp. pec7120 & & 2 \\
\hline Bacillus subtilis & 10 & \\
\hline Clostridium perfringens & & 9 \\
\hline Escherichia coli & 7 & \\
\hline Haemophilus & & 6 \\
\hline \multirow[t]{3}{*}{ Leprospira interrogans } & & $223 \mathrm{~S}$ \\
\hline & & $216 \mathrm{~S}$ \\
\hline & & $15 \mathrm{~S}$ \\
\hline Mycobacterium leprae & 1 & \\
\hline Mycobacterium smagmatis & 2 & \\
\hline \multirow[t]{2}{*}{ Mycoplasma gallisepticum } & & $123 \mathrm{~S}-5 \mathrm{~S}$ \\
\hline & & $116 \mathrm{~S}$ \\
\hline \multirow[t]{2}{*}{ Mycoplasma hypopneumoniae } & & $116 \mathrm{~S}-23 \mathrm{~S}$ \\
\hline & & $15 \mathrm{~S}$ \\
\hline Pirellula marina & & $223 \mathrm{~S}-5 \mathrm{~S}$ \\
\hline Pseudomonas aeruginosa & 4 & $216 \mathrm{~S}$ \\
\hline Streptomyces ambofaciens & 4 & \\
\hline Streptomyces coelicolor & 6 & \\
\hline \multirow[t]{2}{*}{ Thermus thermophilus } & & $223 \mathrm{~S}-5 \mathrm{~S}$ \\
\hline & & $216 \mathrm{~S}$ \\
\hline Vibrio harveyi & & $23 \mathrm{~S}-16 \mathrm{~S}-5 \mathrm{~S}$ \\
\hline
\end{tabular}




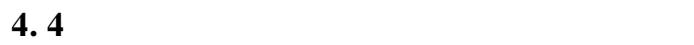

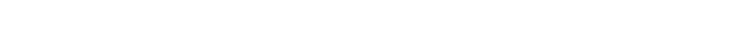


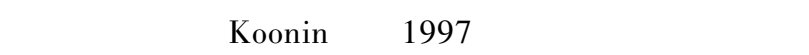

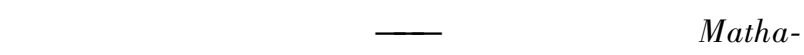

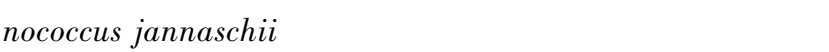

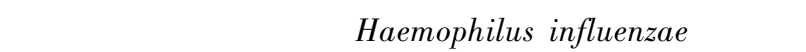

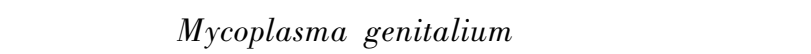

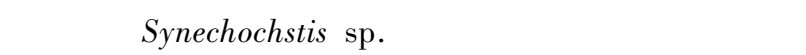

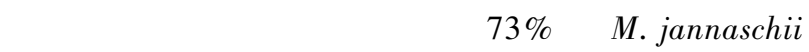

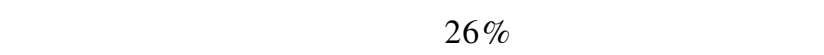

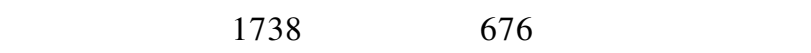

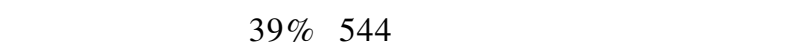

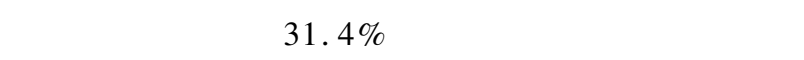

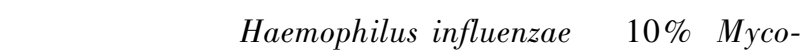
plasma genitalium $\hat{O} / 420 \%$ of Synechochstis sp. Ô $/ 4$

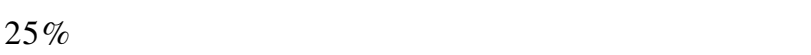

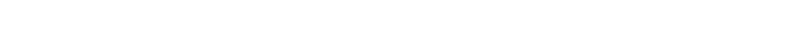

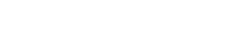

\section{1囟Êøव́}

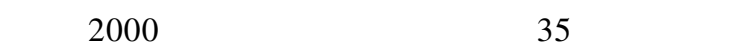

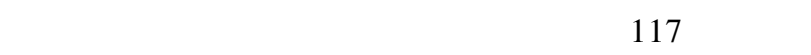

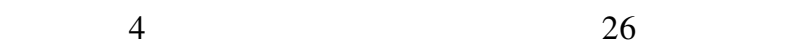

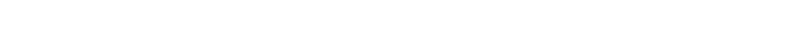

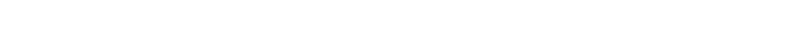

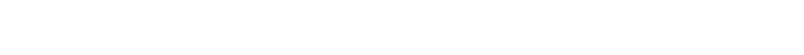

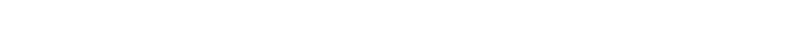

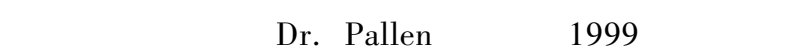

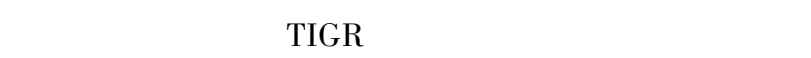

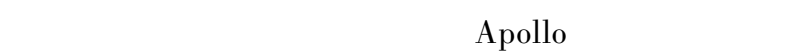

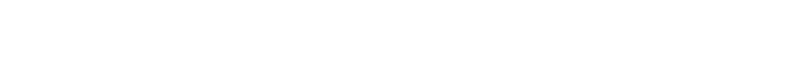

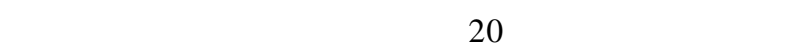

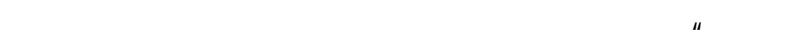

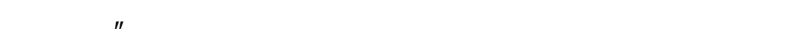

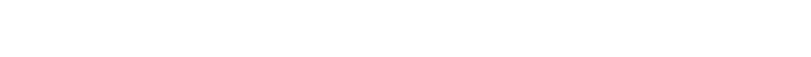

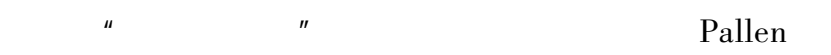

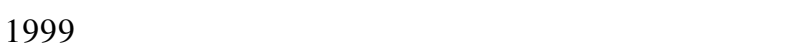

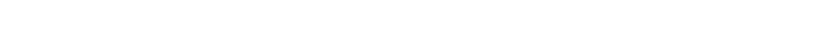

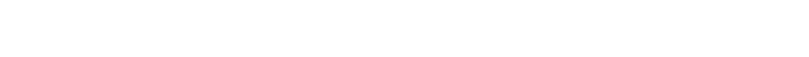

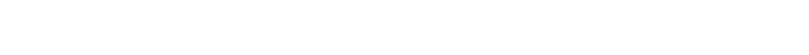

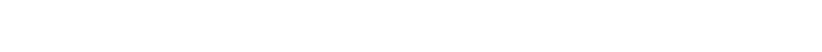

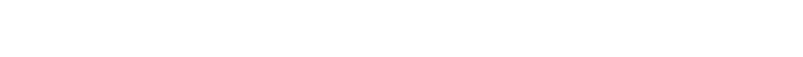

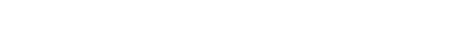

$2 \hat{\imath} \iota^{1} \hat{h} \ddot{A} \mid x$

Amann R I, Ludwig W and Schleifer K H, 1995. Phylogenetic identification and in situ detection of individual microbial cells without cultivation. Microbiology Reviews, 59: 143 169

Brown J R and Doolittle W F, 1997. Archaea and the prokaryote-to-eukaryote transition. Microbiology and Molecular Biology Reviews, 61(4) : $456 \sim 502$

Bull A T, Goodfellow M and Slater J H, 1992. Biodiversity as a source of innovation in biotechnology. Annual Reviews of Microbiology, 40: $219 \sim 252$

Cole S T and Girons I S, 1994. Bacterial genomics. FEMS Microbiology Reviews, 14: 139 160

Garrity G, 2000. Appendix 2: Bergey's manual of systematic bacteriology. In: Madigan M T, Martinko J M and Parker J (eds). Brock Biology of Microorganisms (9th Ed.), Prentice Hall

George and Gerald, 1996. The role of the genome project in determining gene function insights from model organisms. Cell, 86: $521 \sim 529$

Hugenholtz P, Goebel B M and Pace N R, 1998. Mini-review: impact of culture-independent studies on the emerging phylogenetic view of bacterial diversity. Journal of Bacteriolo$g y, \mathbf{1 8 0}(18): 4765 \sim 4774$

Jensen M, Webster J A and Straus N, 1993. Rapid identification of bacteria on the basis of polymerase chain reactionamplified ribosomal DNA spacer polymorphisms. Applied and Environment Microbiology, 59(4) : $945 \sim 952$

Koonin E V, Mushegian A R, Galperin M Y and Walker D R, 1997. Comparison of archaeal and bacterial genomes: computer analysis of protein sequences predicts novel functions and suggests a chimeric origin for the archaea. Molecular Microbiology, 25: $619 \sim 637$

Pallen M J (1999). Micro-Meeting: microbial genomes. Molecular Microbiology, 32(5) : $907 \sim 912$

Stackebrandt E and Geobel B M, 1994. Taxonomic note: a place for DNA-DNA reassociation and 16S rRNA sequence analysis in the present species definition in bacteriology. International Journal of Systematic Bacteriology, 44:846 849

Tiedje J M, 1994. Microbial diversity: of value to whom? ASM News, 60: $524 \sim 525$

Woese C R and Fox G E, 1977. Phylogenetic structure of the prokaryotic domain: the primary kingdoms. Proceedings of National Academy of Sciences, USA, 74: $5088 \sim 5090$

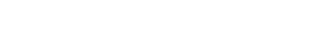

